\title{
TOTAL DOMINATION POLYNOMIALS OF SOME GRAPHS
}

\section{A. R. LATHEESHKUMAR and ANIL KUMAR V.}

Department of Mathematics

St. Mary's College

Sulthan Bathery

Wayanad

Kerala 673592

India

e-mail: latheesby@gmail.com

Department of Mathematics

University of Calicut

Malappuram

Kerala 673635

India

e-mail: anil@uoc.ac.in

\begin{abstract}
A hypergraph is an ordered pair $H=(V, E)$, where $V$ is a finite nonempty set called vertices and $E$ is a collection of subsets of $V$, called hyper edges or simply edges. A subset $T$ of vertices in a hypergraph $H$ is called a vertex cover if $T$ has a nonempty intersection with every edge of $H$. The vertex covering number $\tau(H)$ of $H$ is the minimum size of a vertex cover in $H$. Let $\mathcal{C}(H, i)$ be the family of vertex covering sets of $H$ with cardinality $i$ and let $c(H, i)$ be the cardinality
\end{abstract}

2010 Mathematics Subject Classification: 05C31, 05C69.

Keywords and phrases: total domination, vertex cover, total domination polynomial.

Received November 23, 2016

(C) 2016 Scientific Advances Publishers 
of $\mathcal{C}(H, i)$. The polynomial $\sum_{i=\tau(H)}^{|V(H)|} c(H, i) x^{i}$ is defined as vertex cover polynomial of $H$. For a graph $G=(V, E), H_{G}$ denotes the hypergraph with vertex set $V$ and edge set $\left\{N_{G}(x) \mid x \in V\right\}$. In this paper, we prove that the total domination polynomial of a connected graph $G$ is the vertex cover polynomial of $H_{G}$. Using this result, we determine total domination polynomials of cartesian products of certain classes of graphs.

\section{Introduction}

All graphs considered in this paper are simple and connected unless otherwise stated. Notations and definitions not given here can be found in $[2,5,9]$. A graph is an ordered pair $G=(V(G), E(G))$, where $V(G)$ is a finite nonempty set and $E(G)$ is a collection of 2-point subsets of $V$. The sets $V(G)$ and $E(G)$ are the vertex set and edge set of $G$, respectively. The cartesian product of two graphs $G$ and $H$, denoted $G \square H$, to be the graph with vertex set $V(G) \times V(H)$ and edges between two vertices $\left(a_{1}, b_{1}\right)$ and $\left(a_{2}, b_{2}\right)$ if and only if either $a_{1}=a_{2}$ and $b_{1} b_{2} \in E(H)$ or $b_{1}=b_{2}$ and $a_{1} a_{2} \in E(G)$. The open neighbourhood of a vertex $v \in V(G)$ is $N_{G}(v)=\{u \in V \mid u v \in E(G)\}$. The closed neighbourhood of a vertex $v \in V(G)$ is $N_{G}[v]=N_{G}(v) \cup\{v\}$. If the graph $G$ is clear from the context, we write $N(v)$ and $N[v]$ rather than $N_{G}(v)$ and $N_{G}[v]$. A dominating set, of a graph $G=(V, E)$ with no isolated vertex is a set $S$ of vertices of $G$ such that every vertex in $G \backslash S$ is adjacent to a vertex in $S$. If no proper subset of $S$ is a dominating set of $G$, then $S$ is a minimal dominating set of $G$. The domination number of $G$, denoted by $\gamma(G)$, is the minimum cardinality of a dominating set of $G$. A dominating set of $G$ of cardinality $\gamma(G)$ is called a $\gamma(G)$-set. Let $\mathcal{D}(G, i)$ be the family of dominating sets of $G$ with cardinality $i$ and let $d(G, i)=|\mathcal{D}(G, i)|$. The polynomial $\mathcal{D}(G, x)=\sum_{i=\gamma(G)}^{|V(G)|} d(G, i) x^{i}$ is defined as domination polynomial 
of $G$. A total dominating set, abbreviated TD-set, of a graph $G=(V, E)$ with no isolated vertex is set $S$ of vertices of $G$ such that every vertex of $G$ is adjacent to a vertex in $S$. If no proper subset of $S$ is a TD-set of $G$, then $S$ is a minimal TD-set of $G$. The total domination number of $G$, denoted by $\gamma_{t}(G)$, is the minimum cardinality of a TD-set of $G$. A TD-set of $G$ of cardinality $\gamma_{t}(G)$ is called a $\gamma_{t}(G)$-set. Let $\mathcal{D}_{t}(G, i)$ be the family of total dominating sets of $G$ with cardinality $i$ and let $d_{t}(G, i)=\left|\mathcal{D}_{t}(G, i)\right|$. The polynomial $\mathcal{D}_{t}(G, x)=\sum_{i=\gamma_{t}(G)}^{|V(G)|} d_{t}(G, i) x^{i}$ is defined as total domination polynomial of $G$. A hypergraph $H=(V, E)$ is a finite nonempty set $V=V(H)$ of elements called vertices, together with a finite multiset $E=E(H)$ of subsets of $V$, called hyper edges or simply edges. The order and size of $H$ are $|V|$ and $|E|$, respectively. A k-edge in $H$ is an edge of size $k$. A subset $T$ of vertices in a hypergraph $H$ is a transversal (also called vertex cover) if $T$ has a nonempty intersection with every edge of $H$. The transversal number $\tau(H)$ of $H$ is the minimum size of a transversal in $H$. For further information on hypergraphs refer [4]. Let $\mathcal{C}(H, i)$ be the family of vertex covering sets of $H$ with cardinality $i$ and let $c(H, i)=|\mathcal{C}(H, i)|$. The polynomial $\mathcal{C}(H, x)=\sum_{i=\tau(H)}^{|V(H)|} c(H, i) x^{i}$ is defined as vertex cover polynomial of $H$. For a graph $G=(V, E)$, the $O N H(G)$ or $H_{G}$ is the open neighbourhood hypergraph of $G ; H_{G}=(V, C)$ is the hypergraph with vertex set $V\left(H_{G}\right)=V$ and with edge set $E\left(H_{G}\right)=C=\left\{N_{G}(x) \mid x \in V\right\}$ consisting of the open neighbourhoods of vertices of $V$ in $G$. Let $\Gamma$ be a finite group with identity $e$. Let $S \subseteq \Gamma$ such that $e \notin S$ and $S=S^{-1}$, that is, $S$ is inverse closed. Then the Cayley graph $G=C a y(\Gamma, S)$, is defined as a graph with vertex set $V(G)=\Gamma$ and edge set $E(G)=\left\{(a, b) \mid a b^{-1} \in S\right\}$. 
We need the following theorems to prove the main results of this paper.

Theorem 1.1 ([8]). The ONH of a connected bipartite graph consists of two components, while the ONH of a connected graph that is not bipartite is connected.

Theorem 1.2 ([9]). If $G$ is a graph with no isolated vertex and $H_{G}$ is the ONH of $G$, then $\gamma_{t}(G)=\tau\left(H_{G}\right)$.

Theorem 1.3 ([1]). If a graph $G$ consists of $m$ components $G_{1}, G_{2}, \ldots, G_{m}$, then $D(G, x)=D\left(G_{1}, x\right) \ldots D\left(G_{m}, x\right)$.

Theorem 1.4 ([1]). For every $n \geq 4$,

$$
D\left(P_{n}, x\right)=x\left[D\left(P_{n-1}, x\right)+D\left(P_{n-2}, x\right)+D\left(P_{n-3}, x\right)\right],
$$

with initial values $D\left(P_{1}, x\right)=x, D\left(P_{2}, x\right)=x^{2}+2 x, D\left(P_{3}, x\right)=x^{3}+$ $3 x^{2}+x$

Theorem 1.5 ([1]). For every $n \geq 4$,

$$
D\left(C_{n}, x\right)=x\left[D\left(C_{n-1}, x\right)+D\left(C_{n-2}, x\right)+D\left(C_{n-3}, x\right)\right]
$$

with initial values $D\left(C_{1}, x\right)=x, D\left(C_{2}, x\right)=x^{2}+2 x, D\left(C_{3}, x\right)=x^{3}+$ $3 x^{2}+3 x$.

Theorem 1.6 ([1]). $D\left(K_{m, n}, x\right)=\left((1+x)^{m}-1\right)\left((1+x)^{n}-1\right)+x^{m}+x^{n}$.

Theorem 1.7 ([10]). Let $L_{n}$ be the graph $P_{n} \square K_{2}$. The domination polynomial for $L_{n}$ satisfies the recurrence: $D\left(L_{n}, x\right)=x(x+2) D\left(L_{n-1}, x\right)$ $+x(x+1) D\left(L_{n-2}, x\right)+x^{2}(x+1) D\left(L_{n-3}, x\right)-x^{3} D\left(L_{n-5}, x\right)$ with initial values, 


\begin{tabular}{|c|c|}
\hline$n$ & $P_{n} \square K_{2}$ \\
1 & $x^{2}+2 x$ \\
2 & $x^{4}+4 x^{3}+6 x^{2}$ \\
3 & $x^{6}+6 x^{5}+15 x^{4}+16 x^{3}+3 x^{2}$ \\
4 & $x^{8}+8 x^{7}+28 x^{6}+52 x^{5}+48 x^{5}+47 x^{4}+2 x^{3}$ \\
5 & $x^{10}+10 x^{9}+45 x^{8}+116 x^{7}+178 x^{6}+148 x^{5}+47 x^{4}+2 x^{3}$ \\
6 & $x^{12}+12 x^{1} 1+66 x^{10}+216 x^{9}+453 x^{8}+604 x^{7}+470 x^{6}+168 x^{5}+17 x^{4}$ \\
\hline
\end{tabular}

\section{Main Results}

Theorem 2.1. The total domination polynomial of a connected bipartite graph $G$ is the product of the vertex cover polynomials of the two components of $H_{G}$, while the total domination polynomial of a connected graph that is not bipartite is the vertex cover polynomial of $H_{G}$.

Proof. The proof follows immediately from the definitions of total dominating set of $G$ and vertex cover polynomial of $H_{G}$.

Theorem 2.2. For a bipartite graph $G$,

$$
D_{t}\left(K_{2} \square G, x\right)=[D(G, x)]^{2} .
$$

Proof. Let $X=\left\{x_{1}, x_{2}, x_{3}, \ldots, x_{m}\right\}, Y=\left\{y_{1}, y_{2}, y_{3}, \ldots, y_{n}\right\}$ be the bipartition of $G$. If $V\left(K_{2}\right)=\{a, b\}$, then the bipartition of $K_{2} \square G$ is $\left\{\left(a, x_{1}\right),\left(a, x_{2}\right), \ldots,\left(a, x_{m}\right),\left(b, y_{1}\right),\left(b, y_{2}\right), \ldots,\left(b, y_{n}\right)\right\},\left\{\left(b, x_{1}\right),\left(b, x_{2}\right)\right.$, $\left.\ldots,\left(b, x_{m}\right),\left(a, y_{1}\right),\left(a, y_{2}\right), \ldots,\left(a, y_{n}\right)\right\}$. For $i=1,2, \ldots, m$ and for $j=1,2, \ldots, n$, we have

$$
\begin{aligned}
& N\left(\left(a, x_{i}\right)\right)=\left\{(a, y) / y \sim x_{i} \text { in } G\right\} \cup\left\{\left(b, x_{i}\right)\right\}, \\
& N\left(\left(b, x_{i}\right)\right)=\left\{(b, y) / y \sim x_{i} \text { in } G\right\} \bigcup\left\{\left(a, x_{i}\right)\right\}, \\
& N\left(\left(a, y_{j}\right)\right)=\left\{(a, x) / x \sim y_{j} \text { in } G\right\} \bigcup\left\{\left(b, y_{j}\right)\right\}, \\
& N\left(\left(b, y_{j}\right)\right)=\left\{(b, x) / x \sim y_{j} \text { in } G\right\} \bigcup\left\{\left(a, y_{j}\right)\right\} .
\end{aligned}
$$


Let $G_{1}$ be a graph with bipartition $\left\{\left(b, x_{1}\right),\left(b, x_{2}\right), \ldots,\left(b, x_{m}\right)\right\}$, $\left\{\left(a, y_{1}\right),\left(a, y_{2}\right), \ldots,\left(a, y_{n}\right)\right\}$, such that $\left(b, x_{i}\right) \sim\left(a, y_{j}\right)$ if and only if $\left(a, x_{i}\right) \sim\left(a, y_{j}\right)$ in $K_{2} \square G$. Similarly we construct another graph $G_{2}$ with bipartition $\left\{\left(a, x_{1}\right),\left(a, x_{2}\right), \ldots,\left(a, x_{m}\right)\right\},\left\{\left(b, y_{1}\right),\left(b, y_{2}\right), \ldots,\left(b, y_{n}\right)\right\}$, such that $\left(a, x_{i}\right) \sim\left(b, y_{j}\right)$ if and only if $\left(b, x_{i}\right) \sim\left(b, y_{j}\right)$ in $K_{2} \square G$. It can be observed that both $G_{1}$ and $G_{2}$ is isomorphic to $G$ and a set $N$ is an open neighbourhood of a vertex in $K_{2} \square G$ if and only if $N$ is a closed neighbourhood of a vertex in $G_{1}$ or $G_{2}$. Therefore, a set $S$ is a total dominating set of $K_{2} \square G$ if and only if $S$ is a dominating set of $G_{1} \cup G_{2}$. So,

$$
\begin{aligned}
D_{t}\left(K_{2} \square G, x\right) & =D\left(G_{1} \cup G_{2}, x\right) \\
& =D\left(G_{1}, x\right) \cdot D\left(G_{2}, x\right) \\
& =[D(G, x)]^{2} .
\end{aligned}
$$

This completes the proof.

Corollary 2.3. From Theorem 2.2, we can infer the following results:

(1) $D_{t}\left(K_{2} \square P_{n}, x\right)=\left[D\left(P_{n}, x\right)\right]^{2}$.

(2) $\gamma_{t}\left(K_{2} \square P_{n}\right)=2 \cdot \gamma\left(P_{n}\right)=2 \cdot\left\lceil\frac{n}{3}\right\rceil$.

(3) $D_{t}\left(K_{2} \square C_{2 n}, x\right)=\left[D\left(C_{2 n}, x\right)\right]^{2}$.

(4) $\gamma_{t}\left(K_{2} \square C_{2 n}\right)=2 \cdot \gamma\left(C_{2 n}\right)=2 .\left\lceil\frac{2 n}{3}\right\rceil$.

(5) $D_{t}\left(K_{2} \square K_{m, n}, x\right)=\left[D\left(K_{m, n}, x\right)\right]^{2}$.

(6) $\gamma_{t}\left(K_{2} \square K_{m, n}\right)=4$.

(7) $D_{t}\left(K_{2} \square B_{m, n}, x\right)=\left[D\left(B_{m, n}, x\right)\right]^{2}$, where $B_{m, n}$ is the bi-star graph.

(8) If $T$ is a tree, then $D_{t}\left(K_{2} \square T, x\right)=[D(T, x)]^{2}$. 
Theorem 2.4. A set $S$ is a dominating set of a cycle $C_{n}$ if and only if it has non empty intersection with the set of vertices of all paths of length two in $C_{n}$.

Proof. Let $S$ be a dominating set and $P=u v w$ be a path of length two in $C_{n}$. If $S \cap\{u, v, w\}=\phi$, then the vertex $v$ is not adjacent to any vertex in $S$ and so $S$ cannot be a dominating set of $C_{n}$. The converse is obvious.

Theorem 2.5. If $n$ is odd, then $D_{t}\left(K_{2} \square C_{n}, x\right)=D\left(C_{2 n}, x\right)$.

Proof. Let the vertices of $K_{2} \square C_{n}$ be labelled as shown in Figure 1 .

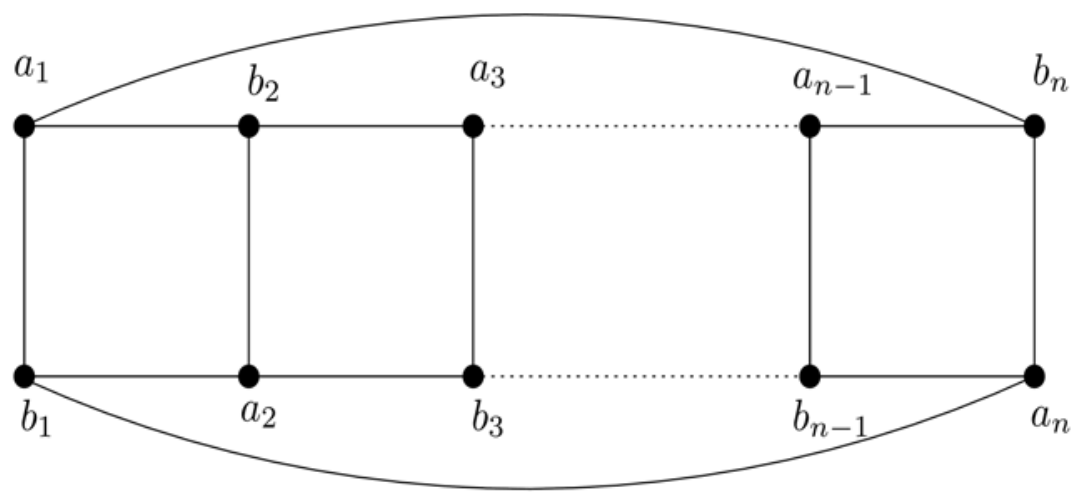

Figure 1. The graph $K_{2} \square C_{n}$.

Let $H_{G}$ be the open neighbourhood hypergraph of $K_{2} \square C_{n}$. Let $C_{2 n}$ be the cycle, $\left(a_{1}, a_{2}, \ldots, a_{n}, b_{1}, b_{2}, \ldots, b_{n}, a_{1}\right)$. It can be observed that if $v \in V\left(K_{2} \square C_{n}\right)$, then the vertices of $N(v)$ form a path of length two in $C_{2 n}$. Also the set of vertices of any path of length two in $C_{2 n}$ is an edge in $H_{G}$. Since $\mathcal{C}\left(C_{2 n}, x\right)=D\left(C_{2 n}, x\right)$, the proof follows from Theorems 2.1 and 2.4 . 
Theorem 2.6. Let $G=\operatorname{Cay}\left(\mathbb{Z}_{n}, S\right)$, where $S=\left\{a, b, b^{-1}\right\}$ is a generating set of $\mathbb{Z}_{n}$ such that $a^{-1}=a$ and $a \notin<b>$. Then,

$$
D_{t}(G, x)= \begin{cases}{\left[D\left(C_{\frac{n}{2}}, x\right)\right]^{2},} & \text { if } n / 2 \text { is even }, \\ D\left(C_{n}, x\right), & \text { otherwise. }\end{cases}
$$

Proof. Here $G \cong K_{2} \square C_{\frac{n}{2}}$. So the proof follows from Corollary 2.3(3) and Theorem 2.5.

Theorem 2.7. Let $G=\operatorname{Cay}\left(\mathbb{Z}_{n}, S\right)$, where $S=\left\{a, b, b^{-1}\right\}$ such that $a^{-1}=a$ and $b$ is a generator of $\mathbb{Z}_{n}$. Then,

$$
D_{t}(G, x)= \begin{cases}D\left(C_{n}, x\right), & \text { if } n \text { is a multiple of } 4, \\ {\left[D\left(C_{\frac{n}{2}}, x\right)\right]^{2},} & \text { otherwise. }\end{cases}
$$

Proof. Since $b$ generates $\mathbb{Z}_{n}, G=\operatorname{Cay}\left(\mathbb{Z}_{n}, S\right)$ can be labelled as shown in Figure 2.

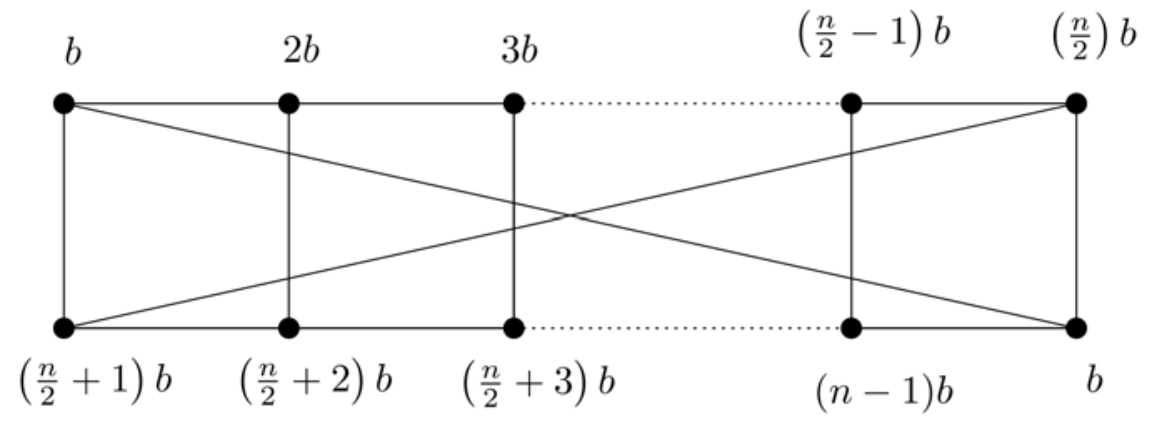

Figure 2. The graph $G=\operatorname{Cay}\left(\mathbb{Z}_{n}, S\right)$.

Case 1. When $n$ is a multiple of 4 .

Consider the cycle $C_{n}=\left(n b,\left(\frac{n}{2}+1\right) b, 2 b,\left(\frac{n}{2}+3\right) b, 4 b,\left(\frac{n}{2}+5\right) b\right.$, $\left.6 b, \ldots,(n-2) b,\left(\frac{n}{2}-1\right) b, n b\right)$. It can be observed that if $v \in V(G)$, then 
the vertices of $N(v)$ form a path of length two in $C_{n}$ and any path of length two in $C_{n}$ is an open neighbourhood of a vertex in $G$. Therefore by Theorem 2.4, $D_{t}(G, x)=D\left(C_{n}, x\right)$.

Case 2. When $n$ is not a multiple of 4 .

In this case the graph $G=\operatorname{Cay}\left(\mathbb{Z}_{n}, S\right)$ is bipartite. Let $X=\{k b / k$ is odd $\}$ and $Y=\{s b / s$ is even $\}$ be the bipartition. Let $H_{X}=(Y,\{N(x) / x \in X\})$ and $H_{Y}=(X,\{N(x) / x \in Y\})$ be the components of the open neighbourhood hypergraph of $G$. Since $H_{X}$ is isomorphic to $H_{Y}$, by Theorem 2.1, $D_{t}(G, x)=\left[\mathcal{C}\left(H_{X}, x\right)\right]^{2}$. Also $E\left(H_{X}\right)=\left\{N(b), N\left(\left(\frac{n}{2}+2\right) b\right)\right.$, $\left.N(3 b), \ldots, N\left(\left(\frac{n}{2}\right) b\right)\right\}=\left\{\left\{n b,\left(\frac{n}{2}+1\right) b, 2 b\right\},\left\{\left(\frac{n}{2}+1\right) b, 2 b,\left(\frac{n}{2}+3\right) b\right\}\right.$, $\left.\left\{2 b,\left(\frac{n}{2}+3\right) b, 4 b\right\}, \ldots,\left\{\left(\frac{n}{2}-1\right) b, n b,\left(\frac{n}{2}+1\right) b\right\}\right\}$.

Consider the cycle, $C_{\frac{n}{2}}=\left(n b,\left(\frac{n}{2}+1\right) b, 2 b,\left(\frac{n}{2}+3\right) b, 4 b, \ldots,\left(\frac{n}{2}-1\right)\right.$ $b, n b)$, with vertex set $Y$. It is obvious that if $v \in Y$, then the vertices of $N(v)$ form a path of length two in $C_{\frac{n}{2}}$. Also any path of length two in $C_{\frac{n}{2}}$ is an open neighbourhood of a vertex in $Y$. Therefore by Theorem 2.4, $\mathcal{C}\left(H_{X}, x\right)=D\left(C_{\frac{n}{2}}, x\right)$. This completes the proof.

Lemma 2.8. If $G$ is an $n-1$ regular bipartite graph, then

$$
\begin{aligned}
D(G, x)= & n x^{2}\left[1+\left(\begin{array}{c}
2 n-2 \\
1
\end{array}\right) x+\left(\begin{array}{c}
2 n-2 \\
2
\end{array}\right) x^{2}+\ldots+\left(\begin{array}{c}
2 n-2 \\
n-3
\end{array}\right) x^{n-1}\right] \\
& +\left[n\left(\begin{array}{c}
2 n-2 \\
n-2
\end{array}\right)+2\right] x^{n}+\left(\begin{array}{c}
2 n \\
n+1
\end{array}\right) x^{n+1}+\left(\begin{array}{c}
2 n \\
n+2
\end{array}\right) x^{n+2}+\ldots+\left(\begin{array}{c}
2 n \\
2 n
\end{array}\right) x^{2 n} .
\end{aligned}
$$


Proof. Let $X=\left\{a_{1}, a_{2}, \ldots, a_{n}\right\}$ and $Y=\left\{b_{1}, b_{2}, \ldots, b_{n}\right\}$ be the bipartition of $G$. Assume that $a_{i}+b_{i}$ for all $i$. If $a_{r}, b_{r} \in S$ for some $r$, then $S$ is a dominating set of $G$. Since there are $n$ pairs, one pair can be selected in $n$ ways. So when $k<n$, the coefficient of $x^{k}$ in $D(G, x)$ is $n\left(\begin{array}{c}2 n-2 \\ k-2\end{array}\right)$. When $k=n$, since $X$ and $Y$ are dominating sets of $G$, the coefficient of $x^{n}$ is $n\left(\begin{array}{c}2 n-2 \\ n-2\end{array}\right)+2$. When $k>n$, any subset of the vertices of $G$ contains a pair $a_{i}, b_{i}$. So the coefficient of $x^{k}$ is $\left(\begin{array}{c}2 n \\ k\end{array}\right)$. This completes the proof.

Theorem 2.9. $D_{t}\left(K_{2} \square K_{n}, x\right)=D(G, x)$, where $G$ is an $n-1$ regular bipartite graph on $2 n$ vertices.

Proof. Let $V\left(K_{2}\right)=\{a, b\}$ and $V\left(K_{n}\right)=\{1,2,3, \ldots, n\}$. Let $A=\{(a, 1),(a, 2), \ldots,(a, n)\} \quad$ and $\quad B=\{(b, 1),(b, 2), \ldots,(b, n)\} . \quad$ Then $V\left(K_{2} \square K_{n}\right)=A \cup B$. Also $N((a, i))=\{(b, i)\} \cup A \backslash\{(a, i)\} \quad$ and $N((b, i))=\{(a, i)\} \cup B \backslash\{(b, i)\}$. We construct an $(n-1)$-regular bipartite graph $G$ in which the open neighbourhoods $N((a, i))$ and $N((b, i))$ are represented as star graphs with root vertices $(b, i)$ and $(a, i)$, respectively.

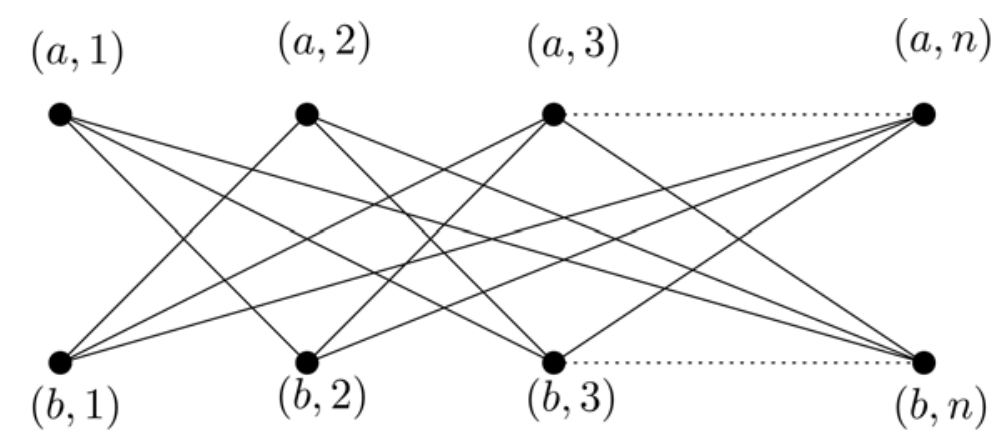

Figure 3. The graph $G$. 
Let $S$ be a dominating set of $G$. Then for all $i$, either $(a, i) \in S$ or $(a, i)$ is adjacent to some vertex in $S$. If $(a, i) \in S$ for all $i$, then $S \cap N$ $((b, i)) \neq \phi$ for all $i$. If $(a, r) \notin S$ for some $r$, then there exists $(b, s) \in S$ for some $s \neq r$. Therefore $S \cap N((b, i)) \neq \phi$ for all $i$. Similarly, we can prove that $S \cap N((a, i)) \neq \phi$ for all $i$. Therefore $S$ is a vertex covering set of $H_{K_{2} \square K_{n}}$. Conversely, let $S$ is a vertex covering set of $H_{K_{2} \square K_{n}}$. Consider a vertex $(a, i)$. If $(a, i) \in S$, then there is nothing to prove. If $(a, i) \notin S$, then $(b, j) \in S$ for some $j \neq i$. So $(a, i) \sim(b, j)$ in $G$. Similarly we can prove the case of $(b, j)$ also. Therefore $S$ is a dominating set of $G$. Thus the result follows from Theorem 2.1.

Next, we determine the total domination polynomials of cartesian product of certain classes of graphs with $C_{4}$.

Theorem 2.10. For a bipartite graph $G$,

$$
D_{t}\left(C_{4} \square G, x\right)=\left[D\left(K_{2} \square G, x\right)\right]^{2} .
$$

Proof. Since the graph $K_{2} \square K_{2} \square G$ is isomorphic to $C_{4} \square G$, the result follows from Theorems 2.1 and 2.2.

Corollary 2.11. From Theorem 2.10, we can infer the following results:

(1) $D_{t}\left(C_{4} \square P_{n}, x\right)=\left[D\left(L_{n}, x\right)\right]^{2}$.

(2) $\gamma_{t}\left(C_{4} \square P_{n}\right)=2\left\lceil\frac{n+1}{2}\right\rceil$.

(3) $D_{t}\left(C_{4} \square C_{2 n}, x\right)=\left[D\left(K_{2} \square C_{2 n}, x\right)\right]^{2}$.

(4) $\gamma_{t}\left(C_{4} \square C_{2 n}\right)= \begin{cases}2 n, & \text { if } n \text { is even, } \\ 2 n+2, & \text { otherwise. }\end{cases}$ 


\section{References}

[1] S. Alikhani and Y. H. Peng, Introduction to Domination Polynomial of a Graph, arXIv: 09052241 v1[math.co] 14 May 2009.

[2] Rangaswami Balakrishnan and K. Ranganathan, A Textbook of Graph Theory, Springer Science \& Business Media, 2012.

[3] B. Chaluvaraju and V. Chaitra, Total domination polynomial of a graph, Journal of Informatics and Mathematical Sciences 6(2) (2014), 87-92.

[4] Ernest J. Cockayne, R. M. Dawes and Stephen T. Hedetniemi, Total domination in graphs, Networks 10(3) (1980), 211-219.

[5] Frank Harary, Graph Theory, 1969.

[6] Teresa W. Haynes, Stephen T. Hedetniemi and Peter J. Slater, Domination in graphs: Advanced topics, Volume 209 of Monographs and Textbooks in Pure and Applied Mathematics, 1998.

[7] Teresa W. Haynes, Stephen T. Hedetniemi and Peter J. Slater, Fundamentals of Domination in Graphs, CRC Press, 1998.

[8] Michael A. Henning and Anders Yeo, Hypergraphs with large transversal number and with edge sizes at least 3, Journal of Graph Theory 59(4) (2008), 326-348.

[9] Michael A. Henning and Anders Yeo, Total Domination in Graphs, New York, Springer, 2013.

[10] Tomer Kotek, James Preen and Peter Tittmann, Domination polynomials of graph products, arXiv preprint arXiv: (2013), 1305-1475.

[11] A. Vijayan and S. Sanal Kumar, On total domination sets and polynomials of cycles, International Journal of Mathematical Archive (IJMA) 3(4) (2012). 